\title{
The time course of phonological and orthographic processing of acronyms in reading: Evidence from eye movements
}

\author{
TIMOTHY J. SLATTERY, ALEXANDER POLLATSEK, and KEITH RAYNER \\ University of Massachusetts, Amherst, Massachusetts
}

\begin{abstract}
The present experiment presented acronyms preceded by either $a$ or $a n$ in a sentence. The key stimuli were acronyms that begin with a consonant such as $F$, whose letter name begins with a vowel sound, or with the vowel $U$, whose letter name begins with a consonant sound. The durations of first fixations were influenced solely by phonology: For example, $a$ FBI caused as much disruption (relative to an $F B I$ ) as did an CIA (relative to a CIA). Somewhat later processing (most notably go-past time and total time) was influenced by orthography as well, as combinations such as an FBI had longer processing times than did combinations such as $a$ CIA. Although the initial coding of visual stimuli must proceed from an orthographic code to a phonological one, these data indicate that the phonological representation of the acronym is the primary initial code that makes contact with the article that precedes it and suggest that the initial contact with the sentence context is phonological.
\end{abstract}

Many recent studies on the role of phonology in skilled reading have indicated that phonology enters into the process of word identification early (Rayner, Pollatsek, \& Binder, 1998). That is, phonological codes are obtained from a word even before it is fixated (Chace, Rayner, \& Well, 2005; Henderson, Dixon, Petersen, Twilley, \& Ferreira, 1995; Miellet \& Sparrow, 2004; Pollatsek, Lesch, Morris, \& Rayner, 1992) and during the first $50 \mathrm{msec}$ of the initial fixation on the word (Lee, Binder, Kim, Pollatsek, \& Rayner, 1999). Surprisingly, the activation of phonological codes occurs almost as rapidly in Chinese, where an assembly of the phonological code from graphemes smaller than a syllable is not possible (Liu, Inhoff, Ye, \& Wu, 2002; Pollatsek, Tan, \& Rayner, 2000; Tsai, Lee, Tzeng, Hung, \& Yen, 2004). One set of utterances in which the activation of phonological codes has not been examined in English, however, comprises acronyms such as FBI. ${ }^{1}$ Such sequences of letters are interesting, because they are not pronounced in the usual way. That is, they do not usually form pronounceable utterances according to the rules of spelling-to-sound correspondence. (Our discussion assumes, unless explicitly stated otherwise, that we are excluding acronyms such as NATO and UNICEF, which are pronounced as if they were words.) Acronyms are interesting in the context of silent reading, because they provide an unusual opportunity, and one that has not been previously explored, to contrast the effects of pho-

This research was supported by Grant HD26765 from the National Institutes of Health. We thank David Plaut and two anonymous reviewers for their helpful comments on the article. Correspondence regarding this article should be sent to T. J. Slattery, Department of Psychology, University of Massachusetts, Amherst, MA 01003 (e-mail: tslatter@ psych.umass.edu). nology and orthography. So, for example, the phonological code for the acronym FBI is presumably "eff bee eye" (our experiment will present data consistent with this notion), and although it begins with a vowel sound, its first letter is an orthographic consonant.

In the present study, we examine how orthography and phonology are used during the silent reading of acronyms. We also employ a different technique from that in the studies cited above; there is no display change or priming stimulus in this study. Instead, phonological coding is probed with the indefinite articles, $a$ and $a$, using the fact that the initial sounds of the names of some letters are different from how they sound when they begin a normal word. As noted above, the sound of $F$, when pronounced as a letter "eff," begins with a vowel sound even though $F$ is a consonant (and pronounced as a consonant in words). Conversely, the sound of $U$, when pronounced as a letter, begins with a semivowel sound even though $U$ is a vowel. ${ }^{2}$ Thus, phonological coding can be probed by examining whether readers have greater difficulty when reading $a$ $F B I$ in a sentence than when reading an $F B I$. That is, if the early (and primary) coding of $F B I$ is orthographic, then $a$ $F B I$ should be easier to process, whereas if the early (and primary) coding of $F B I$ is a letter-by-letter phonological code, then an FBI should be easier to process. Logically, of course, orthographic coding must precede phonological coding when stimuli are presented visually. However, the higher level cognitive systems involved in reading may not be operating on this orthographic representation primarily, but rather on the phonological representation that derives from it. As we discuss orthographic and phonological effects here, we will be referring to the representation of the lexical item that is communicating with the other cognitive systems involved in reading. 
It is not clear that there are "rules" for deciding whether to assign $a$ or $a n$ to such acronyms, or, if there are, whether the typical reader is aware of them. Although some advocate that usages such as an FBI are correct, spell checkers on word processors accept either $a F B I$ or an $F B I$ or they sometimes mark an FBI as an error and accept $a F B I .{ }^{3}$ To assess whether or not printed text follows a phonological rule for such cases, we conducted a corpus analysis of acronyms using a preliminary release of the American National Corpus. The initial search was for all acronyms that were preceded by an indefinite article; then the search results were edited to remove cases of acronyms that are read by the usual spelling-to-sound correspondences (e.g., NATO). The results of this search, presented in Table 1, clearly show that there is a great deal of uncertainty over which indefinite article to use when the acronym begins with a consonant that has a vowel sounding name. Also worth noting is that, even if a usage is correct, an incorrect version may actually be processed more efficiently (at least in the initial processing stages). For example, for readers of German, the initial processing of compound words actually proceeds better when there are spaces between the constituents, even though the spacing is illegal because compounds in German, by rule, are always written without spaces (Inhoff, Radach, \& Heller, 2000). Thus, examining the initial processing of such article acronym units is interesting regardless of "correct usage."

In this experiment, we employed four types of acronyms: (1) ones beginning with consonants such as FBI, where the initial phoneme of the name of the first letter was a vowel; (2) ones beginning with consonants such as CIA, where the initial phoneme of the name of the first letter was a consonant; (3) ones beginning with vowels such as USA, where the initial phoneme of the name of the first letter was a consonant; and (4) ones beginning with vowels such as IRS, where the initial phoneme of the name of the first letter was a vowel. In addition, each of the four types of acronyms was preceded by either $a$ or an. The employment of conditions (2) and (4) provided a baseline for the effect in conditions (1) and (3). That is, since both the orthographic and phonological information in conditions (2) and (4) pointed to the same "correct" use of the article, this should provide an estimate of the maximal effect for providing a mismatching article. Examining the size and direction of the "article effect" for conditions (1) and (3) should indicate whether orthographic or phonological coding predominates and might even indicate that one type of coding might not be relevant at all.

Table 1

Frequency of Article Assignment in the American National Corpus

\begin{tabular}{lccc}
\hline $\begin{array}{c}\text { Type of } \\
\text { Acronym }\end{array}$ & Total & $\begin{array}{c}\text { Phonologically } \\
\text { Consistent }\end{array}$ & $\begin{array}{c}\text { \% Consistent With } \\
\text { Phonological Rule }\end{array}$ \\
\hline FBI/USA & 433 & 286 & 66.1 \\
CIA/IRS & 574 & 572 & 99.7 \\
\hline
\end{tabular}

\section{METHOD}

\section{Participants}

The participants were 34 native English speakers from the University of Massachusetts community. ${ }^{4}$ Each participant received either course credit or money for his or her participation.

\section{Apparatus}

Eye movements were recorded by a Fourward Technologies Dual Purkinje Eyetracker, which has a resolution of less than $10^{\prime}$ of arc; the output is linear over the angle subtended by a line of text. The eyetracker was interfaced with a Pentium computer. The computer was also interfaced with a 15 -in. NEC MultiSync 4FG color monitor on which the sentences were presented. The display was $61 \mathrm{~cm}$ from the participant's eye, and four characters equaled $1^{\circ}$ of visual angle. Viewing was binocular, but eye movements were recorded from the participant's right eye. A bitebar was used to eliminate head movements during the experiment.

\section{Materials}

The target stimuli were acronyms preceded by an indefinite article. The acronyms were two to five letters in length (mean $=3.07$ ) and were chosen so that most could not be pronounced as words; all of them are standardly read aloud by reading the letter names in sequence. All were embedded in simple sentences in which the article and the acronym were preceded by an average of 6.9 words and followed by an average of 3.3 words. Each acronym appeared in one sentence, but there were two versions of the sentence: One with $a$ preceding the target word, and one with an preceding the target word, as in the following example.

1. The kidnapper was caught by a FBI undercover agent.

2. The kidnapper was caught by an FBI undercover agent.

There were 30 target acronyms: 20 began with a consonant and 10 with a vowel (the acronyms used are presented in the Appendix). Half of the acronyms in each set began with a letter whose name began with a vowel sound (e.g., FBI, IRS) and the other half began with a letter whose name began with a consonant or semivowel sound (e.g., CIA, USA). ${ }^{5}$ There were two lists in which the conditions were approximately counterbalanced so that no participant saw any acronym more than once.

\section{Procedure}

When participants arrived for the experiment, they read a description of the procedure. A bitebar was then prepared, and the procedure was explained verbally in more detail. The eyetracker was then calibrated (which takes about $5 \mathrm{~min}$ ). They were then told that they would be given sentences to read and were asked to read each sentence for normal comprehension. On each trial, the participant was instructed to fixate a small box on the left of the screen, and a single sentence (on a single line of text) was presented; the participant pressed a button when he/she had read the sentence. To ensure thorough reading, a comprehension question was presented after one third of the sentences, and the participants responded to these questions by pressing a button.

\section{RESULTS}

To simplify exposition, we will refer to pairs such as an FBI, an IRS, a CIA, and a USA as phonologically consistent, and to the same acronyms with the opposite article as phonologically inconsistent. Similarly, we will refer to pairs such as a FBI, a CIA, an IRS, and an USA as orthographically consistent, and to these acronyms with the opposite article as orthographically inconsistent. The mea- 
sures of fixation time are presented below in the order that documents the time course of the effects of phonological and orthographic consistency. The earliest measure that we report is first fixation duration (the duration of the first fixation on the acronym), and the measure that includes somewhat later processing is gaze duration (the sum of the durations of all fixations on the acronym prior to movement to another word). (Both measures are conditional on there being a fixation on the word.) Later measures will be discussed in more detail below. The acronym (including the space before it) was selected as the target region for first-pass processing rather than the region that included both article and acronym, because the size of the latter region depended on whether the article was $a$ or an. (One could try to deal with this problem by including a space when the article was $a$; however, the regions would still be different, because one would contain one fewer letter.) Although such a small target region is potentially problematic because it could be skipped frequently (and thus result in sparse fixation duration data), as will be seen below, the skipping rates were not particularly high.

Below, our analyses are collapsed across the acronyms that start with a vowel and those that start with a consonant. We did this for two reasons. First, a combined analysis that treated this variable explicitly had many empty cells because there were few observations per cell for the vowel-initial acronyms, and thus about a third of the participants were excluded from the analysis. (The same problem occurred in analysis of the vowel-initial acronyms separately.) Second, when each set is analyzed separately, any pure orthographic effect is confounded with whether the acronym is preceded by $a$ or $a n$; this is counterbalanced in the combined analysis. All of the eye-tracking measures are presented in Table 2.

\section{First Fixation Duration}

This measure, as indicated above, taps the earliest processing of the acronym, and thus may be the most interesting. There was a $19-\mathrm{msec}$ main effect of phonological consistency on this measure that was highly reliable $\left[F_{1}(1,32)=11.76, p<.002 ; F_{2}(1,26)=15.75, p<\right.$ $.001] .{ }^{6}$ Perhaps of equal interest is that there was no indication of an orthographic consistency effect; the phonological consistency effect was the same for the acronyms whose phonology agreed with orthography $(19 \mathrm{msec})$ and for the acronyms whose phonology disagreed with orthography $(19 \mathrm{msec})$. The main effect of orthographic consistency (independent of phonology) was half the difference between these difference scores, so that the best estimate of the orthographic consistency effect is $0 \mathrm{msec} .^{7}$

\section{Gaze Duration}

The pattern for gaze duration was somewhat different, with the suggestion of an effect of orthographic consistency. The 19-msec phonological consistency effect on gaze durations was significant $\left[F_{1}(1,32)=6.77, p<.025\right.$; $\left.F_{2}(1,26)=9.02, p<.01\right]$, and the 13 -msec orthographic consistency effect was marginally significant in the participant analysis $\left[F_{1}(1,32)=3.25, p<.10 ; F_{2}(1,26)=\right.$ $2.43, p<.20]$.

\section{Measures of Later Processing}

A measure of slightly later processing is go-past time, which is the sum of all fixations between when the target region is entered and when it is exited to the right; it thus includes the durations of all regressions to the left of the target region and any refixation on the target region before it is exited. This measure showed effects of both phonological and orthographic consistency that were significant in one analysis and marginally significant in the other. There was a 24-msec main effect of phonological consistency $\left[F_{1}(1,32)=2.93, p<.10 ; F_{2}(1,26)=5.90\right.$, $p<.025]$ and a 42-msec main effect of orthographic consistency $\left[F_{1}(1,32)=15.79, p<.001 ; F_{2}(1,26)=3.59\right.$, $p<.10] .{ }^{8}$ There was little effect of either phonological or orthographic consistency on the next measure of downstream processing, the spillover duration, which is the mean duration of the first fixation after leaving the target region to the right. The phonological consistency effect was $1 \mathrm{msec}$ and the orthographic consistency effect was $-1 \mathrm{msec}(F \mathrm{~s}<1)$. Another measure of later processing is second pass time on the target word (i.e., fixation time due to regressions back to the target word). This is the mean time spent on the target word after it has been exited to the right. (When there is no regression back to the target word, second pass time is counted as $0 \mathrm{msec}$.) There were only suggestions of orthographic and phonological consistency effects on this measure; the phonological consistency effect was $12 \mathrm{msec}\left[F_{1}(1,32)=1.18, p>.20 ; F_{2}(1,26)=\right.$ $2.56, p<.20]$, and the orthographic consistency effect

Table 2

Eye Movement Measures (Fixation Time, in Milliseconds), With Percentages Fixated, for the Target Region

\begin{tabular}{lcccccccc}
\hline & & \multicolumn{9}{c}{ Fixation Time } & & \\
Consistency & & First & Gaze & Go & Spillover & Second & Total & \% \\
Condition & Examples & Fixation & Duration & Past & Duration & Pass & Time & Fixated \\
\hline Both & a CIA/an IRS & 266 & 275 & 301 & 266 & 44 & 318 & 71.9 \\
Neither & an CIA/a IRS & 285 & 307 & 367 & 266 & 64 & 364 & 72.4 \\
Difference & & 19 & 32 & 66 & 0 & 20 & 46 & 0.5 \\
Phonology & an FBI/a USA & 255 & 269 & 349 & 268 & 54 & 278 & 68.7 \\
Orthography & a FBI/an USA & 274 & 275 & 330 & 270 & 58 & 285 & 70.0 \\
Difference & & 19 & 6 & -19 & 2 & 4 & 7 & 1.3 \\
\hline
\end{tabular}


was $8 \mathrm{msec}\left(F_{\mathrm{S}}<1\right)$. A global measure that includes all fixations on the target word is total time. On this measure, both the 27-msec phonological consistency effect and the 20-msec orthographic consistency effect were significant $\left[F_{1}(1,32)=6.08, p<.025 ; F_{2}(1,26)=8.31, p<.01 ;\right.$ and $F_{1}(1,32)=4.82, p<.05 ; F_{2}(1,26)=5.58, p<.05$, respectively].

In sum, there was a clear phonological consistency effect on the earliest measure (first fixation duration), but no orthographic consistency effect. In contrast, there were indications of an orthographic consistency effect as well in later measures, especially on total time. To make sure that none of the fixation duration effects above were artifacts of a differential probability of a first-pass fixation on the target region, we also analyzed this measure, and (see Table 2) there was virtually no indication of either an orthographic or a phonological consistency effect on the probability of fixating the acronym $(F \mathrm{~s}<1)$.

\section{DISCUSSION}

In early processing, the sole cue on which the agreement between the article and acronym is based is the sound of the initial letter name rather than whether the initial letter is a vowel or consonant. That is, there was no evidence that orthographic agreement between the article and the acronym had a facilitative effect on first fixation duration. This leaves open the question, however, of whether the observed "phonological effect" was due to conversion of the acronym to a phonological code or to some confounding variable (such as a rule or frequency of usage).

As indicated earlier, the evidence from the corpus analyses in Table 1 is that there is no uniform "rule" guiding the assignment of an to acronyms such as FBI. However, to determine whether participants, on reflection, thought that there was a phonological rule governing the assignment of the indefinite article to such acronyms, 17 undergraduates at the University of Massachusetts (who had not participated in the experiment) were given the sentences that had been used in the experiment with the indefinite articles removed, and they were asked to fill in the appropriate indirect articles. The results of this offline study (presented in Table 3) are consistent with the corpus search discussed earlier. Of these 17 participants, 5 seemed to apply a phonological rule, 4 appeared to use an orthographic rule, and the remaining 8 were not consistent in their assignment of indirect articles. These data indicate that people from our participant pool have no strong biases about whether to assign $a$ or an to such acronyms.

These rating data do not necessarily rule out a frequency explanation of our phonological agreement effect on first fixation duration, especially if one posits that the effect occurs at an unconscious level. That is, one could argue that even though combinations like an FBI are not used all the time, they are somewhat more frequent than combinations like $a F B I$, and that the more often readers see a word (or a combination of words) in print, the easier it is for them to process these words. Such a frequency argument,
Table 3

Completions in the Article Assignment Task

$\%$ Consistent With a Phonological Rule

\begin{tabular}{cc} 
Type of Acronym & a Phonological Rule \\
\hline FBI/USA & 67.8 \\
CIA/IRS & 96.1 \\
\hline
\end{tabular}

however, seems quite implausible. First, if the relative frequency of the use of $a$ versus $a n$ was the reason for an apparent phonological agreement effect, one would expect that the effect size would differ among the items in the FBI/USA condition, with a stronger phonological agreement effect the greater the relative frequency of using the phonologically appropriate article. Unfortunately, many of the acronyms were quite infrequently preceded by an indefinite article in our corpus search, so that using the relative frequency of $a$ versus $a n$ in this corpus for a particular acronym seemed like a very unreliable measure of this bias. Instead, we used the relative frequency with which the phonologically appropriate article was used in our offline task as our best estimate of a possible frequency of co-occurrence bias and calculated the correlation between this estimate of the bias and the effect size in first fixation duration (over the items in the FBI/USA condition). Contrary to the prediction of such a hypothesis, the correlation was close to zero and in fact slightly negative $(r=-.106$, $p>$.7). Moreover, this small correlation was not due to a restricted range effect, because the bias value ranged from $23.5 \%$ to $94.1 \%$ with a mean of $66.3 \%$. Second, perhaps an even stronger argument against a frequency explanation is that the phonological agreement effect for acronyms such as FBI and USA in first fixation duration was exactly the same as that for acronyms such as CIA and IRS (19 msec), even though the former were preceded by the phonologically inconsistent article $33.9 \%$ of the time in our corpus search, whereas the latter were preceded by the phonologically inconsistent article only $0.3 \%$ of the time. Thus, although the frequency argument would predict that all of the groups of acronyms should show a "phonological" effect, the argument should also predict that the effect is significantly larger for acronyms such as CIA and IRS that are virtually completely consistent in the assignment of the article than for acronyms such as FBI and USA, where the usage was fairly inconsistent.

Moreover, this lack of a difference between the orthographically consistent and orthographically inconsistent acronyms on first fixation duration is not likely to be due to a lack of statistical power, for we found such a difference in later measures of processing - most reliably in the go-past and total time measures. These differences between orthographically consistent and orthographically inconsistent acronyms indicate that the ease of processing the article-acronym pair is not completely determined by phonological principles. These later effects, however, could be consistent with a "true" orthographic effect (i.e., to some analytic orthographic process occurring at the time of encoding the acronym), or they could simply 
reflect the more consistent usage of the phonologically consistent article for acronyms such as CIA and IRS (the frequency explanation developed above).

Thus, the effects observed in the present study appear to reflect two processing stages. The first depends on the phonological match between the article and the initial letter, and the second is primarily dependent on the orthographic match between the article and the initial letter. This leaves open the question of exactly what the cause is for a difference between match and mismatch in each of these stages. We offer the following hypothesis for the early phonological effect observed in the first fixation duration data. When the acronym FBI is preceded by $a$, it biases the initial phonological coding incorrectly by guiding the reader to process the $F$ as if it were pronounced as in normal reading (the $F$ in Friday). Although this would be the appropriate bias for acronyms such as NATO, it is inappropriate for an acronym like FBI and thus causes disruption. (A similar argument obviously applies to an acronym like USA.) This hypothesis is quite consistent with the fact that the phonological consistency effect is early for both types of acronyms. That is, it is unlikely that there is an orthographic analysis rapid enough to determine that the phonological code for the acronym is a sequence of letter names or a pronunciation of the acronym as if it were a word. Note that USA or NIH could be pronounced as words yet are not, and that NHTSA (National Highway Traffic Safety Administration) is called "nitsa." For an acronym like CIA, the mechanism would have to be different. That is, regardless of the type of acronym that starts with $\mathrm{C}$, there is a phonological mismatch between $a n$ and the acronym. The data indicate that the disruption caused by this phonological mismatch also occurs early. In contrast, the effect of an orthographic consistency effect that is not redundant with the phonological consistency effect would be expected only to appear later, possibly only after the acronym is identified and determined by the relative frequency of occurrence of the two articles with that acronym.

In sum, just as prior research has indicated that phonological coding is activated early in the processing of printed words in text (e.g., Lee et al., 1999; Pollatsek et al., 1992), the present research indicates that such coding is also activated early in the processing of acronyms whose phonological code is a sequence of letter names. In addition, the present data indicate that this phonological information is not only activated quickly, but also quickly integrated with the prior phonological information in the text.

\section{REFERENCES}

Chace, K. H., Rayner, K., \& Well, A. D. (2005). Eye movements and phonological parafoveal preview: Effects of reading skill. Canadian Journal of Experimental Psychology, 59, 209-217.

Henderson, J. M., Dixon, P., Petersen, A., Twilley, L. C., \& Ferreira, F. (1995). Evidence for the use of phonological representations during transsaccadic word recognition. Journal of Experimental Psychology: Human Perception \& Performance, 21, 82-97.

InHOFF, A. W., RADACH, R., \& Heller, D. (2000). Complex compounds in German: Interword spaces facilitate segmentation but hinder assignment of meaning. Journal of Memory \& Language, 42, 23-50.

Lee, Y.-A., Binder, K. S., Kim, J.-O., Pollatsek, A., \& Rayner, K. (1999). Activation of phonological codes during eye fixations in reading. Journal of Experimental Psychology: Human Perception \& Performance, 25, 948-964.

Liu, W., Inhoff, A. W., Ye, Y., \& Wu, C. (2002). Use of parafoveally visible characters during the reading of Chinese sentences. Journal of Experimental Psychology: Human Perception \& Performance, 28, 1213-1227.

Miellet, S., \& Sparrow, L. (2004). Phonological codes are assembled before word fixation: Evidence from boundary paradigm in sentence reading. Brain \& Language, 90, 299-310.

Pollatsek, A., Lesch, M., Morris, R. K., \& Rayner, K. (1992). Phonological codes are used in integrating information across saccades in word identification and reading. Journal of Experimental Psychology: Human Perception \& Performance, 18, 148-162.

Pollatsek, A., Tan, L.-H., \& Rayner, K. (2000). The role of phonological codes in integrating information across saccadic eye movements in Chinese character identification. Journal of Experimental Psychology: Human Perception \& Performance, 26, 607-633.

Rayner, K., Pollatsek, A., \& Binder, K. S. (1998). Phonological codes and eye movements in reading. Journal of Experimental Psychology: Learning, Memory, \& Cognition, 24, 476-497.

Tsai, J., Lee, C., Tzeng, O. J. L., Hung, D. L., \& Yen, N. (2004). Use of phonological codes for Chinese characters: Evidence from processing of parafoveal preview when reading sentences. Brain \& Language, 91, 235-244.

\section{NOTES}

1. The formal definition of acronym appears to differ from dictionary to dictionary. Some reserve the term for such letter sequences that spell a word, such as NATO or snafu, but others agree with our usage and consider items such as $F B I$ as acronyms. We will use the term in the latter, broader sense.

2. The initial pronunciation of a word-initial $u$ is not invariably a vowel sound as in union, and thus such words are preceded by $a$. The only other deviation from the rule that words beginning with vowels are preceded by $a n$ and words beginning with consonants are preceded by $a$ is words beginning with silent $h$, such as an honor. (British usage carries this even further, however, with examples such as an historic.)

3. See www.edufind.com/english/grammar/Determiners3.cfm.

4. Participants were asked at the end of the session what they noticed about the materials. Somewhat surprisingly, only 2 seemed to be aware that there were disagreements between the articles and acronyms even after fairly direct questions such as "Did you notice anything strange about the sentences with acronyms?" Some other participants indicated that they were unfamiliar with one or two of the acronyms used, and some indicated having noticed that many of the acronyms began with the letter $U$. The 2 who were aware of the disagreements between the articles and acronyms were not included in the analyses here, because their fixation times may have included making mental notes of the disagreements and, more generally, trying to figure out what the experiment was about. Thus, the data here were from participants whose knowledge of a "rule" (if any) was not sufficiently strong to cause them to consciously notice violations of it.

5 . The reason that there were only five stimuli in each of the vowel conditions was that the only vowel whose letter name begins with a consonant is $U$, and we were worried that seeing too many $U$ acronyms might cause the participants to develop special strategies in the experiment.

6. Counterbalancing group was included in all the analyses.

7. That is, the main effect of orthography would be equal to the average of conditions exemplified by an CIA, an FBI, a USA, and a IRS, minus the average of conditions exemplified by a CIA, a FBI, an USA, and an IRS.

8 . The numbers reported in the text and in the table are from the participants analysis. The pattern in the go-past measure was different in the items analysis, where the phonological consistency effect was bigger than the orthographic consistency effect. 
APPENDIX

\begin{tabular}{ll}
\multicolumn{2}{c}{ APPENDIX } \\
\hline FBI & CIA \\
SOS & PBS \\
MTV & KGB \\
MRI & BMW \\
LSU & DEA \\
NBA & CEO \\
NFL & VIP \\
NBC & PO \\
HBO & CBS \\
FDA & BLT \\
USA & IRS \\
UPS & EPA \\
UNLV & ASPCA \\
UV & ESPN \\
USDA & APB \\
\hline
\end{tabular}

(Manuscript received January 12, 2005;

revision accepted for publication September 28, 2005.) 